\title{
THE “PSYCHOSOMATIC FAMILY” RECONSIDERED: DIABETES IN CONTEXT
}

\author{
James C. Coyne Barbara J. Anderson \\ University of Michigan Medical School
}

\begin{abstract}
Minuchin, Rosman and Baker's (1978) Psychosomatic Families is widely cited as a demonstration that the physiological disturbance of some diabetic patients serves a function in their families. We found that the original data did not provide such a demonstration. We examined the psychosomatic family model in light of recent developments in the study and treatment of diabetes. We concluded that the model decontextualizes the family and assigns to it characteristics that are more appropriately seen as reflections of the disease process, the family coping tasks this entails, and the nature of the family's relationship with the health care system. The need for new open-systems models of the family's role in diabetes is discussed.
\end{abstract}

Minuchin, Rosman and Baker's (1978) Psychosomatic Families is truly a classic work, and it will long be recognized as an important first step for the field. The provocative book is one of the most frequently cited ones from the field of family therapy (Forman, 1986), in large part because of the attention it has received, not only in the family journals, but in pediatrics, psychiatry, psychology, and social work. The claim that ongoing family interaction had been shown to influence free fatty acid (FFA) levels in the blood of diabetic children legitimized further exploration of links from family functioning to such life-threatening problems as anorexia and poorly controlled diabetes and asthma. The book also provided an important theoretical statement, and a decade after its appearance, it retains its position as the most clearly articulated theoretical framework for what Weakland (1977) has called family somatics-our understanding of how the individual's interactions with the family impact on physical health and vice versa. Finally, the book provided impressive outcome data from the successful family treatment of anorexia nervosa, as well as provocative clinical examples, and it has done much to widen the appeal not only of Minuchin's structural approach to therapy, but the full range of family therapies.

It should be noted that although the book is frequently cited as a convincing demonstration of both the immediate effect of family interaction on physiology and the homeostatic function of the patient's physiological disturbance in the family, the actual data available in the book were preliminary and summarized in an impressionistic manner. It was stated that a more complete and formal presentation would be forthcoming. A decade later, the publications from the Philadelphia Child Guidance Clinic continue to make strong claims about what these data purportedly demonstrate (Sar-

James C. Coyne, PhD, is Associate Professor of Family Practice and Psychiatry, University of Michigan Medical School, 1018 Fuller Street, Box 0708, Ann Arbor, MI 48109.

Barbara J. Anderson, PhD, is Assistant Research Scientist, Diabetes Research and Training Center and Department of Pediatrics, University of Michigan Medical School, Ann Arbor, MI 48109. 
gent, 1985), but there has still not been a full presentation of the data, and there have been no published replications of the study. Subsequent citations and discussions of the data presented in Psychosomatic Families generally convey a sense that the data were so compelling that they stand on their own. Further, the theoretical statement and clinical observations developed in the book have largely been accepted without refinement or even critical scrutiny. The theoretical model developed in the book was described as generally applicable to a full range of health problems, and that is how the model has been widely interpreted.

Since the publication of Psychosomatic Families, family systems theory has increasingly gained adherents in the field of family medicine (Doherty \& Baird, 1983), and there is now a journal, Family Systems Medicine, devoted to the interface of family systems and health. Yet, as one reviewer (Dakof, 1987) has noted, research and theory on families and somatic health still remains stuck at the starting gate. A cursory glance at Social Science Citations Index or Science Citations Index will demonstrate that none of this more recent work has achieved the attention or credibility of Psychosomatic Families. Furthermore, with very few exceptions (e.g., Campbell, 1987), this work generally accepts the conclusions of Minuchin et al. (1978) without the slightest demur. The kind of critical thinking needed for the vigorous development of the field is too seldom in evidence.

A critical reexamination of Psychosomatic Families is timely, not because such a decade-old work should be held to current standards, but because its conclusions continue to be accepted as definitive. Despite its immense influence, the book has failed to provide sufficient momentum for the field to move beyond it. In terms of the development of the field, the ideas presented in the book have served, not as a rung on a ladder, but a place to rest. We will show that having initially succeeded in widening the range of considerations in serious health problems to include the family, the model presented in the book now serves to constrain our attempts to understand and treat diabetes and other health problems in the context of the family.

We are focusing our reexamination of Minuchin et al.'s (1978) model specifically as it applies to insulin-dependent diabetes mellitus (IDDM) for a number of reasons. First, the effectiveness of the therapeutic approach associated with the psychosomatic family model was demonstrated with anorexia nervosa, but it was only for IDDM that the important link was claimed between observations of the family in an experimental task situation and the children's FFA levels. Minuchin et al. (1978) noted the potential significance of such a demonstration:

In our research we have been able to document the power of family rules by measuring their effect on the FFA in the bloodstream of the diabetic. The soft data of transactional patterns have been given scientific confirmation (p. 330).

Further, the argument for the general model of psychosomatic families critically depended on the data from the diabetic children:

FFA is not related to an asthmatic attack; indeed, there are many physiological reasons to believe that free fatty acids are peripheral to the events through which emotional arousal is mediated into an asthmatic attack. Similarly, free fatty acids do not lie in any straight line between the psyche and the somatic event in anorexia nervosa. It is only in diabetes that this direct link exists, and it is in this group that there is physiological evidence providing strong support for our hypotheses of psychosomatic disease (p. 45).

Consistent with this, Minuchin et al. noted, "physiological derangement could not be demonstrated in either psychosomatic asthmatics or the anorectics" (p. 49).

A second set of reasons for focusing on diabetes lies in the complex relationship between diabetes and family functioning. Diabetes has a significant impact on the
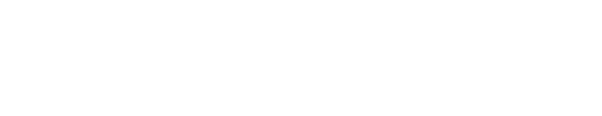
family, and conversely, family functioning has been found to be related to biomedical outcomes (Anderson \& Auslander, 1980). The treatment of IDDM is characterized by an almost immediate transfer of responsibility from health care professionals to the family. In addition to progressive long-term health risks, individuals with IDDM must maintain a fine balance between high and low blood sugar episodes and often suffer the disabling symptoms of abnormal metabolic states. To attempt to achieve well controlled blood sugar levels, a complex treatment plan must be carried out 24-hours-a-day which involves the most basic components of daily life-food, physical activity, finances and time. Family members are required to make clinical judgments that affect metabolic functioning, and the diabetic regimen may have a profound impact upon well established family routines.

Thus, treatment planning often complicates normal tasks of family living and even the simplest of family experiences, and it requires major readjustments by the entire family. Because of this complex relationship between the family and control of IDDM, a family systems approach is particularly applicable to this illness. Indeed, Psychosomatic Families is often cited in the diabetes literature as the authoritative source for understanding the role of the family in diabetes, and more general discussions in the diabetes and pediatric literatures sometimes limit their citations of the role of the family in chronic illness to this one book (e.g., Baum \& Kinmonth, 1985).

However, as we will see, much of the research accumulating concerning both the nature of diabetes and the role of the family is incompatible or not readily accommodated by the psychosomatic family model as it stands. For instance, there has been progress in our understanding of adolescents with brittle diabetes. These adolescents have previously unexplained, large changes in blood glucose concentration, sometimes to the point that severe hypoglycemic and hyperglycemic episodes prevent them from maintaining a normal lifestyle (Schade, Drumm, Duckworth \& Eaton, 1985). On the basis of new knowledge about counter-regulatory hormonal activity and insulin resistance during puberty, Minuchin et al.'s (1978) notion of the psychosomatic diabetic would have to be substantially modified and greatly restricted in its usage, and at least some phenomena previously attributed to family interaction might better be seen in terms of the nature of the disease process.

In other instances, important developments in the family approach to the management of diabetes have not received the attention they deserve because of the dominance of the psychosomatic family model. As an example of this, there have been well controlled studies of interventions with the families of adolescents in poor control that disregard the model's assumptions concerning the functional significance of the diabetic patient's problems for the family's organization (Golden, Herrold \& Orr, 1985). By their success, these interventions seemingly contradict these assumptions of the model. Importantly, such interventions concentrate on the patient and family's role in the patient receiving insufficient insulin rather than their presumed role in instigating or maintaining the patient's level of arousal. Many difficulties in the management of IDDM can be traced to ignorance, misinformation, and families' well meant, but miscarried, problem solving. Understanding and remedying these difficulties requires attention, not only to the family as an isolated unit, but to the health care system with which every family with a chronically ill child has had, and will continue to have, multiple interactions.

Psychosomatic Families was important in calling attention to the interconnectedness of the family and the management of chronic health problems. Yet, ironically, the psychosomatic family model now serves to promote misunderstanding by decontextualizing the families of children with IDDM, ignoring their interconnection with the health care system. Phenomena that are more appropriately seen in terms of difficulties in interactions between families and health care systems are misconstructed as structural defects of families.

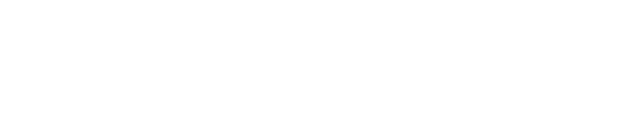


At this point in the development of the field, we still do not have a minimally adequate model of the multileveled system of the diabetic child in the context of the family and the family in the larger context of the health care system. To remedy this problem, we need to first come to terms with the limitations of the psychosomatic family model. We will begin our reconsideration of it by examining the original FFA data as they are presented in Psychosomatic Families.

\section{THE FAMILY INTERACTION AND FFA DATA: A CLOSER LOOK}

In their second chapter, Minuchin et al. (1978) describe the evolution of their work from early interviews of diabetic children and clinical observations of young asthmatic, diabetic, and anorectic patients to more systematic studies of the patients and their families participating in structured tasks such as construction of a color-form design and discussion of various topics, and finally, to the involvement of the families in stressful diagnostic interviews. The interview procedure involved precipitating and exacerbating parental conflict in an effort to study both the patients' role in parental conflict and the physiological effects of the conflict on the child and parents. Only the data from this interaction task are presented in any detail, and over the past decade these data have been viewed as a compelling demonstration of the functional connection between family interaction and individual physiology.

The sample providing the observational data consisted of 45 families. Nine of them had a child considered to have "psychosomatic diabetes," 11 had a young anorectic patient, and 10 had a child with asthma. The control groups consisted of 7 families with "normal or nonpsychosomatic" diabetes and 8 families with diabetics whose illness was under good medical control, but who had been referred for behavioral problems. However, the FFA results were limited to the three groups with diabetic children, with 7 of the families of the normal and 8 of the behavior-problem diabetic children serving as controls for 7 of the families of children with "psychosomatic diabetes."

The families participated in a three-phase interview, and heparin locks allowed the drawing of blood during both the interview and a followup rest period. FFA levels were determined from these blood samples. In the first phase of the interview, the parents were seated in a room with the child observing and listening through a one-way mirror. The parents discussed an issue about which there had been some disagreement, with the parents of the control diabetic children tending to focus on marital and parental issues and the parents of the "psychosomatic diabetic" children talking more about their concerns for their ill child. After a half hour, an experimenter entered the room and attempted deliberately to exacerbate the conflict revealed in the first phase of the interview. The details that are given are sparse, but apparently the experimenter took sides and pressed one or both spouses on issues that had emerged. After another half hour, the child was brought into the room, and the parents and the child were asked to assist each other in a discussion of change. The interview was then terminated after another half hour, and the family was taken to an office where final blood samples were drawn.

On pages 45 and 46, Minuchin et al. (1978) provide a discussion of the FFA data from three groups of diabetic children: "psychosomatic," behavioral-problem, and normal. Reference is made to Figure 3 on page 46, yet that figure is captioned: "Changes in FFA of anorectic [emphasis added] children during family interview." The description in the text suggests that it is the figure that is erroneously labeled. Assuming it is the text that is correct, what the figure seems to show is that the psychosomatic diabetic children began getting upset (i.e., their FFA levels increased from baseline) when they observed the parents' discussion of a family problem from behind a one-way mirror. They continued to do so when the interviewer deliberately exacerbated the disagreement
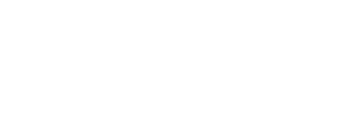
between the parents, and when they, themselves, entered the room with the parents and were instructed by the interviewer to help the parents decide how to change. The arousal also continued when they were taken with their parents to a room to relax. In contrast, the normal-diabetic children decreased in FFA level until they entered the interview room, at which time they returned to their baseline FFA level, only to decrease again when they were sent with their families to another room to relax. The diabetic children with behavioral problems basically showed the same pattern as the normal diabetic children.

The text goes on to state that: "The physiological evidence also supported the hypothesis that the psychosomatic symptom plays a role in family homeostasis," citing Figure 4. The data are described as demonstrating a crossover effect, such that the most aroused parent became less so after the child entered the room, and the child's FFA level continued to rise:

The physiological measurement showed the presence of the child decreased the parent's emotional arousal, at the cost of a continued rise in the child's arousal, propelling him toward disease. The sustained arousal of FFA during the recovery period attested to the maintenance of the pattern in the face of unresolved family conflict (p. 46).

It is difficult to see how the data gave rise to this interpretation. Figure 4 is labeled: "Medians of parent with higher FFA response and index patient," and thus, the data points for each type of family - those with normal, behavior-problem, or psychosomatic diabetic children-represent information from one parent and one child, and very likely not a parent and a child from the same family. Thus, it appears that Minuchin et al.'s (1978) analyses do not preserve the family as the unit of analysis. In general, this manner of presenting data is not standard, and limits the kinds of inferences that can be made. Without knowing the statistical significance of group differences or even means or standard deviations, it is hazardous to rely on visual inspection of these data. Apparent differences may be less than what could be expected by chance, and this is particularly likely to be the case with such a small sample size.

However, it does appear that FFA of the median index patient steadily increased from start of experiment through the designated rest period. The FFA level of the median higher parent dropped at the end of period two, which marked not only the entrance of the child, but the end of the experimenter's efforts to exacerbate the conflict between the parents. Which of these two changes was most influential or whether the size of the change was significant is not clear. In either case, the evidence of a linkage between parent and child FFA levels is weak, at best.

In short, as they are presented, the data simply do not offer strong support for the psychosomatic family model, or the homeostatic function of poorly controlled diabetes. Recall that unlike the other children, the psychosomatic diabetic children were in the midst of recurring medical crises, possibly life-threatening, and they were most likely to be the object of their parents' discussion than the other children were. The content of this discussion, thus, differed from that of the other parents, and was more personally threatening to the children. We can only speculate as to how the experimenter exacerbated the parental conflict, but given the authors' strong commitment to a functional interpretation of the children's problems with metabolic control, it is not unreasonable to assume that these parents were dealing with a qualitatively different and more upsetting provocation from the experimenter than the other parents were. Certainly, most parents would become upset when a professional implied or stated directly that their child's life-threatening medical crisis is in some way tied to their unacknowledged marital problems.

Probably, the most parsimonious interpretation of the FFA data is that the psychosomatic children became upset as their parents discussed the seriousness of their situ- 
ation, and they continued to do so when asked to assist their parents in discussing how to deal with them. As measured by FFA levels, their parents became less upset when the experimenter stopped antagonizing them and allowed them to involve the children in a discussion of the child's serious medical problems. The psychosomatic diabetic children's FFA levels may have risen dramatically, but this occurred in an uninterrupted, straightforward fashion, starting when they were observing through the one-way mirror, rather than in the complex way postulated by the psychosomatic family model. Examined closely, the data are less compelling than the text that accompanies them, but it is the text, and not the data, themselves, that has captured the attention of the field.

\section{"PSYCHOSOMATIC" PATTERNS OF FAMILY FUNCTIONING}

Based on observations and impressions during family assessments, diagnostic interviews and family therapy sessions, Minuchin and his colleagues defined four patterns of family functioning as characteristic of psychosomatic families: (a) enmeshment, (b) overprotectiveness, (c) rigidity, and (d) lack of conflict resolution. They report that no one of these patterns was singly responsible for triggering psychosomatic symptoms in the ill child, but rather, "the cluster of transactional patterns was felt to be characteristic of a family process that encourages somatization" (p. 30). We will first define each of these terms briefly, using the authors' explanations and examples. Subsequently, we will argue that rather than "encouraging somatization," these very interdependent family characteristics can plausibly be viewed as consequences of a family besieged by repeated, life-threatening medical emergencies (i.e., diabetic ketoacidosis).

The first characteristic of the psychosomatic family, enmeshment, describes an overly involved and overly responsive quality in family relationships, in which members intrude on each others' thoughts and feelings as well as on communications within the family. Overprotectiveness, the second characteristic of the psychosomatic family, is reflected in the excessive nurturing and sensitivity of family members to one another's distress.

Rigidity, or resistance to any form of change, is the third trait of the psychosomatic family. The family has difficulty in accommodating to the growth of autonomy in developing children, as well as to any external stimuli which stress the family's framework Rigid families "typically represent themselves as normal and untroubled, except for the one child's medical problem" (p. 31). The fourth defining feature of the psychosomatic family-lack of conflict resolution-appears to be a cumulative consequence of the three previously described traits. Conflicts remain chronically unresolved and constantly threaten the family's equilibrium.

This portrait of the psychosomatic family suggests that its constellation of problems-lack of boundaries, overinvolvement, and difficulty with change and conflictboth encourages and is maintained by psychosomatic symptoms in the diabetic child. While vividly described, these four characteristics of family functioning are not discussed with any reference to coping with the stresses of illness. The psychosomatic diabetic child is seen as a "conflict defuser" (p. 22), playing an active role in maladaptive family interactions. Likewise, the parents are said to use the ill child to detour around conflict, especially marital issues. Yet, while conflict is central to the model of the psychosomatic family, it is de-contextualized from the real conflicts such as negotiating symptom monitoring, compliance with treatment, or the adequacy of health care which face diabetic children and families, especially those with repeated, acute medical emergencies.

From our vantage point, the "enmeshment" and "overprotectiveness" Minuchin finds in psychosomatic families could be viewed as inevitable responses to repeated metabolic crises in a child, rather than deficiencies which "encourage somatization."
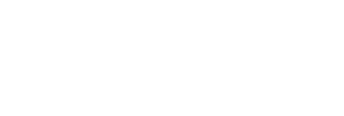
Close parental scrutiny is sometimes warranted by the diabetic child's age, disease duration, health status, and regimen complexity. A high degree of involvement is sometimes unavoidable, as parents struggle with unpredictable short-term illnesis crises, all the while continuing to carry out multiple daily treatment requirements.

Psychosomatic families are described as rigid and heavily committed to the status quo.

lssues that threaten change, such as negotiations over individual autonomy, are not allowed to surface to the point where thay can be explored. Even when coming into therapy, these families typically represent themselves as normal and untroubled, except for the one child's medical problems. They deny any need for change in the family. (p. 31)

This is a plausible clinical picture, but it is difficult to falsify. It invites the inference that a family that doubts the relationship between diabetes and the family and that sees the lack of overt conflict as an achievement, not a deficiency, is, thus, revealing itself to be a "psychosomatic family." In many instances, such a description could reflect a family's appropriate resistance to a professional's theory-driven insistence that a child's health crises are benefiting, or caused, or even needed, by the family.

Thus, in the final chapter of Psychosomatic Families, "Psychotherapy for a Small Planet," Minuchin's small planet is quite small, indeed, from the vantage point of the present. It encompassed the family and its "interconnectedness" but not the health care delivery contexts with which families with a chronically ill child interact and which provide an important arena for family intervention.

\section{BRITTLE AND UNSTABLE DIABETES: AN UPDATED VIEW}

Minuchin et al.'s (1978) findings are often cited without regard for the unusual and ill-defined sample of "psychosomatic diabetic" children on whom the data are based. Important variables such as age and stage of development, sex, and disease duration, which affect both parent and child behavior as well as physiological variables, are not reported. In fact, surprisingly little physiological information is provided. Minuchin et al. simply pointed out that the diagnosis of "psychosomatic" was made by the pediatrician, "who indicated that there was no organic or physiological reason for the difficulty of medical management" (p. 35).

It should be noted that although most diabetic children seldom, if ever, require hospitalization, a minority have occasional or recurring bouts of acute ketoacidosis, despite competent medical care (Macgillivray, Bruck \& Voorhes, 1981). Over the past decade, researchers have identified factors that can potentially destabilize metabolic control and routinely need to be assessed in children with recurrent control problems. These include such variables as level of endogeneous insulin production, insulin antibody levels, and two areas especially relevant to Minuchin's research, counter-regulatory hormonal activity and the insulin resistance that occurs during puberty.

Recent research has shown that a variety of stresses in humans provoke the counterregulatory hormones-catecholamine, cortisol, glucagon, and growth hormone-all of which antagonize the action of insulin and promote the breakdown of triglycerides to fatty acids. Although these "stress hormones" do not have precisely the same effects throughout carbohydrate metabolism, all can be regarded as antagonistic to insulin, and work either directly or indirectly to raise blood sugar levels. The intensity and character of counter-regulatory responses can be highly individual (Craig, 1981; Macgillivray et al., 1981). Therefore, differences in susceptibility to ketoacidosis among persons with insulin-dependent diabetes, likewise, may be quite individual and may depend, in part, on different stress hormones response patterns. 
Furthermore, the use of free fatty acid levels as the sole marker of stress, must be questioned. Since free fatty acid levels are a by-product of activity of the stress hormones, they were used as a marker of stress by Minuchin et al. in the "stressful" diagnostic interview. However, a recent report by Kemmer, Bisping, Steingruber, Baar, Hardtmann, Schlaghecke \& Berger (1986) indicated that free fatty acid levels did not change under experimental stress conditions for diabetic individuals in good control, those in poor control, or nondiabetic adults, raising questions about the validity of free fatty acid as a measure of stress.

There is another line of research which also makes us more cautious about the current clinical application of the data reported in Psychosomatic Families. In a recent study focused on the impact of puberty on metabolism, Amiel, Sherwin, Simonson, Lauritano \& Tamborlane (1986) reported that insulin action is impaired for diabetic and nondiabetic adolescents during puberty as compared with prepubertal children or with adults. They concluded that the combined adverse effects of puberty and diabetes on insulin action may help to explain why stable blood sugar control is so difficult to achieve in some adolescent patients: "One can envision a vicious cycle in which the puberty-related reduction in insulin sensitivity leads to hyperglycemia, which leads in turn to further resistance and frustrates attempts to maintain compliance in these difficult cases" (p. 219). In summary, new research progress on counterregulation and metabolic disruptions during puberty, broaden, considerably, the potential explanations for recurrent control problems in diabetic children and should temper current tendencies to hypothesize single causes such as family stress for repeated metabolic control problems.

\section{FAMILY FUNCTIONING, DIABETES, AND FAMILY INTERVENTION}

A lengthy nontechnical report on Minuchin's work, published by the National Institute of Mental Health, "Psychosomatic Diabetic Children and their Families" (Segal, 1977), reveals an emphasis on the family as the perpetrator of metabolic problems that has continued to the present day. This popular report, while acknowledging the causal role of patterns of family conflict, disregards the fact that repeated medical crises may prompt tense family conversations focused on the ill child: "... the sickly diabetic appeared to be inextricably and pathologically enmeshed in their families' currents of conflict" (p. 5). In addition, we suggest that interpretations of the Minuchin et al. (1978) data, such as the following, have tended to foster a punitive approach toward individual family members:

“. . . the youngsters' FFA levels rose dramatically as they became involved in their parent's tensions. At the same time, the parents' FFA levels were dramatically reduced. In today's vernacular, the adults had "laid it on" their children. (p. 8)

The implications for intervention are clear:

There is little wonder, then, that all of these acutely ill children fare better when they are removed from the family scene. But whisking sick children away from their parents, Dr. Minuchin is quick to emphasize, is at best a stopgap measure. His ultimate goal is to treat not the child, but the family - to essentially reorganize family patterns. (p. 8)

Must families radically reorganize to insure metabolic stability? Recent intervention research with families struggling with repeated hospitalizations and metabolic crises have indicated that changing basic family interactions is not always prerequisite to successful metabolic outcomes. As we have noted, most diabetic children seldom require repeated hospitalizations for hyperglycemia. Yet, a minority have recurring acute diabetic ketoacidosis (Orr, Golden, Myers \& Marrero, 1983). For this identifiable group of children and adolescents, a cycle of dysfunction develops early, with medical 
emergencies and family crises becoming closely intertwined (White, Kolman, Wexler, Polin \& Winter, 1984). There is a general lack of services for these families within the health care system, and the individual or family therapy which is often recommended is simply economically unfeasible or unacceptable to a family already feeling burdened with the diagnosis of a chronic physical illness. To complicate matters, there is convincing evidence that both younger and older diabetic children, and their parents, often lack basic diabetes knowledge and management skills (Johnson, Silverstein, Rosenbloom, Carter \& Cunningham, 1986; Ingersoll, Orr, Herrold \& Golden, 1986). It is reasonable to assume that these potentially remediable deficiencies contribute to erratic and deteriorating glucose control in some children and families.

In an important study with children and adolescents who had repeated episodes of diabetic ketoacidosis, Golden and colleagues (1985) published a carefully controlled study evaluating the effectiveness of a hierarchical or step-wise intervention approach. This intervention began with a comprehensive biomedical evaluation, educational assessment and skill training in insulin administration and adjustment for children with a history of repeated hospitalizations and their families. Secondly, aggressive efforts were taken to insure that insulin was consistently administered. If the child or adolescent was not able to take this responsibility, a family member was enrolled. If no family member could be counted on, community resources such as visiting nurses or school personnel were engaged to help insure that a consistent insulin schedule was maintained.

Diabetic ketoacidosis was defined by the investigators as "preventable," and thus, failing to insure insulin administration was seen as medical neglect. The issue was not seen as one of the family being blamed for the occurrence of the patient's problems, but of giving them the tools for solving these problems and asking that they assume responsibility for the problems' solutions or to cede this responsibility to the community. Of 35 children, 2 were placed in foster care on this basis. Therapy was recommended in $90 \%$ of the cases, but fewer than half of the families followed through with this recommendation. However, recurrent diabetic ketoacidosis was eliminated in 31 of the 35 families. In addition to reduced hospitalization, metabolic control improved significantly after the intervention. Furthermore, there were no differences in outcomes between families who did or did not participate in therapy. Golden et al.'s research underscores the importance of integrating educational, psychosocial, and biomedical interventions with this high-risk group. Yet, it also raises troubling questions about whether Minuchin et al.'s (1978) concerns about structure, function, and homeostasis are distractions from a consideration of simple, pragmatic solutions to these children and adolescents' health problems.

The role of the health care system in contributing to the adaptation of diabetic children and their families has been systematically ignored in the literature (Anderson \& Auslander, 1980). Most physicians and diabetes clinics are not routinely equipped to intervene in this cycle of metabolic and psychosocial deterioration displayed in certain families, nor are health care providers trained to search for the range of biopsychosocial factors that impinge on compliance and on metabolic control (Sulway, Tupling, Webb \& Harris, 1980; Surwit, Feinglos \& Scovern, 1983). Taken together with documented educational deficiencies in diabetes information and management skills, it is clear that the focus of responsibility and blame for metabolic functioning, as well as the focus for intervention, extends beyond the "psychosomatic family" to include the broader context of primary health care.

\section{SUMMARY AND A LOOK TOWARD FUTURE MODELS}

A close examination of the data presented in Minuchin et al. suggests that they fail to yield a convincing demonstration of the homeostatic role of poorly controlled diabetes 
in the family. Our review has focused specifically on diabetes, but these data have assumed a crucial role in more general arguments that symptoms serve functions in families. It is important that we begin asking what empirical evidence exists for this widely and strongly held view, and reconsider it if such evidence is not forthcoming. As we have seen, the assumption that physical symptoms serve functions has, at times, led to an adversarial view of families of patients with chronic health problems. Further, such an assumption leaves us ill prepared for the apparent success of intervention programs that simply ignore the functional significance of problems in adherence and metabolic control.

Clinically, the assumptions of the psychosomatic model may take on an unfortunate self-confirmatory quality when casually applied to the families of diabetic patients. The clinician who treats these families as adversaries may find validation in their response and miss the resources that the families potentially have to offer. Further, the model's prescription that these families require radical reorganization simply cannot be implemented in many settings, and it is understandable that many families refuse to accept it.

One might argue that successful interventions with the families of poorly controlled diabetic patients either reorganize the family's involvement in the patient's diabetes or insulate the patient's diabetes management from the adverse effects of the family, and in that sense these interventions are "structural" and consistent with the psychosomatic family model. However, at least as described by Golden et al. (1985), it appears that these interventions ignore the crucial assumption of functionality in the maintenance of the family homeostasis and involve a more straightforward and diabetes care-specific approach to these families than the psychosomatic family model prescribes.

While the psychosomatic family model was an important first step for the field, recognizing its limitations must be its next. Earlier psychosomatic models of the diabetic individual have now been discarded because of their inaccurate predictions concerning the psychological characteristics of such persons and their lack of practical utility for physicians and patients attempting to manage the disease (Surwit, Scovern \& Feinglos, 1982). Even a cursory review of the current literature suggests that, broadly applied, the psychosomatic model of the families of diabetic children is similarly outmoded and inadequate as a guide for understanding and intervening in the families of diabetic patients. A more adequate model of diabetes in the family will have to accommodate better both individual differences in the disease process and how they shape the manner in which the family presents itself, as well as the influence on the family of the nature of its contacts with the health care system. In many instances, the psychosomatic family model misconstrues the influence of these factors as structural defects of families.

The psychosomatic family model cautioned us that what was previously seen as a feature of the individual patient is more appropriately viewed as a feature of the individual in a particular family. We suggest that any successors to the model should aid us in recognizing, also, how apparent features of the family, when it is viewed out of context, are more appropriately construed as features of a family in contact with a particular disease process and in crucial ways shaped by its relationship to the health care system. The successor models should involve greater sensitivity to the family as an open system, in interaction with biomedical and institutional factors that may powerfully influence how the family reorganizes and functions. Further, such models should encourage a "principle of charity" (Coyne \& Segal, 1982) in approaching the families of diabetic patients in poor control, allowing for the possibility that misinformation, well meant but miscarried problem-solving, and various types of interfering metabolic factors may be the primary sources of difficulties, rather than leaping to the conclusion that these families need to have problems.
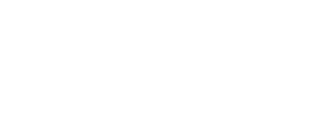


\section{REFERENCES}

Amiel, S. A., Sherwin, R. S, Simonson, D. D., Lauritano, A. A. \& Tamborlane, W. V. (1986). Impaired insulin action during puberty: A contibutory factor to poor glycemic control in adolescents with diabetes. New England Journal of Medicine, 315, 215-219.

Anderson, B. J. \& Auslander, W. A. (1980). Research on diabetes management and the family: A critique. Diabetes Care, 3, 696-702.

Baum, J. D. \& Kinmonth, A. L. (Eds.). (1985). Care of the child with diabetes. London: Churchill Livingstone.

Campbell, T. L. (1987). Family's impact on health: A critical review and annotated bibliography (DHHS Publication No. ADM 87-1461). Washington, DC: U.S. Government Printing Office.

Coyne, J. C. \& Segal, L. (1982). A brief, strategic interactional approach to psychotherapy. In J. Anchin \& D. Kiesler (Eds.), Handbook of interpersonal psychotherapy. New York: Pergamon.

Craig, O. (1981). Childhood diabetes and its management. (2nd edition). Boston: Butterworth.

Dakof, G. A. (1987). At the starting gate: Research and theory on families and somatic health. Journal of Family Psychology, 1, 135-141.

Doherty, W. J. \& Baird, M. A. (1983). Family therapy and family medicine. New York: Guilford.

Forman, B. D. (1986). Citation classics in family therapy. Journal of Marital and Family Therapy, 12, 97-100.

Golden, M. P., Herrold, A. J., and Orr, D. P. (1985). An approach to prevention of recurrent diabetic ketoacidosis in the pediatric population. Journal of Pediatrics, 107, 195-200.

Ingersoll, G. M., Orr, D. P., Herrold, A. J., \& Golden, M. P. (1986). Cognitive maturity and selfmanagement among adolescents with insulin-dependents diabetics mellitus. Journal of Pediatrics, $108,620-623$.

Johnson, S. B., Silverstein, J., Rosenbloom, A., Carter, R. \& Cunningham, W. (1986). Assessing daily management in childhood diabetes. Health Psychology, 5, 545-564.

Kemmer, F. W., Bisping, R., Steingruber, H. J., Baar, H., Hardtmann, F., Schlaghecke, R. \& Berger, M. (1986). Psychosocial stress and metabolic control in patients with Type I diabetes mellitus. New England Journal of Medicine, 314, 1078-1084.

Macgillivray, M. H., Bruck, E. \& Voorhes, M. L. (1981). Acute diabetic ketoacidosis in children: Role of the stress hormones. Pediatric Research, 15, 99-106.

Minuchin, S., Rosman, B. L. \& Baker, L. (1978). Psychosomatic families: Anorexia nervosa in context. Cambridge, MA: Harvard University Press.

Orr, D. P., Golden, M. P., Myers, G. L. \& Marrero, D. G. (1983). Characteristics of adolescents with unstable diabetes referred to a tertiary care center. Diabetes Care, 6, 170-175.

Sargent, J, (1985). Juvenile diabetes mellitus and the family. In P. I. Ahmed \& N. Ahmed (Eds.), Coping with juvenile diabetes. Springfield, IL.: Charles Thomas.

Segal, J. (1977). Psychosomatic diabetic children and their families. Rockville, MD: National Institute of Mental Health, DHEW Publication No. (ADM) 77-477.

Schade, D. S., Drumm, D. A., Duckworth, W. P. \& Eaton, R. P. (1985). The etiology of incapacitating, brittle diabetes. Diabetes Care, $8,12-20$.

Sulway, M., Tupling, H., Webb, K. \& Harris, G. (1980). New techniques for changing compliance in diabetes. Diabetes Care, 3, 108-111.

Surwit, R., Feinglos, M. N. \& Scovern, A. W. (1983). Diabetes and behavior: A paradigm for health psychology. American Psychologist, 38, 255-263.

Surwit, R., Scovern, A. \& Feinglos, M. N. (1982). The Role of behavior in diabetes. Diabetes Care, $5,337-341$.

White, K., Kolman, M. L., Wexler, P., Polin, G. \& Winter, R. J. (1984). Unstable diabetes and unstable families: A psychosocial evaluation of diabetic children with recurrent ketoacidosis. Pediatrics, 73, 749-755.

Weakland, J. (1977). Family somatics: A neglected edge. Family Process, 16, 263-272. 